\title{
ON A LENGTH PRESERVING CURVE FLOW
}

\author{
LI MA, ANQIANG ZHU
}

\begin{abstract}
In this paper, we consider a new length preserving curve flow for convex curves in the plane. We show that the global flow exists, the area of the region bounded by the evolving curve is increasing, and the evolving curve converges to the circle in $C^{\infty}$ topology as $t \rightarrow \infty$.
\end{abstract}

\section{INTRODUCTION}

In this paper, we study a nature evolution non-local flow for convex curves in the plane. This flow preserves the length of curves. We shall obtain the entropy estimate and integral estimates for the evolution to get a global flow. We remark that this kind of technique was used in [7, where they studied the curve shortening flow. Curve shortening flow has been studied extensively in the last few decades (see [7] and [12] for background and more references). It can be showed that the convexity of curves along the curve shortening flow is preserved and the curves become more and more circular before they collapse to a point. Since then, other flows for curves have also been proposed. One may see B.Andrews's papers (see for example, [1]) and Tsai's papers [17] for other kind of flows for curves. One may see [10] for higher dimension flows for hyper-surfaces. As showed by M.Gage [6], some non-local flow for convex curves are also very interesting. In a very recent paper 16, S.L.Pan and J.N.Yang consider a very interesting length preserving curve flow for convex curves in the plane of the form

$$
\frac{\partial}{\partial t} \gamma(t)=\left(\frac{L}{2 \pi}-k^{-1}\right) N,
$$

where $L, N$, and $k$ are the length, unit normal vector,and the curvature of the curve $\gamma(t)$ respectively. They have proved that the convex plane curve will become more and more circular and converges to circle in the $c^{\infty}$ sense. It is interesting to study curve flow which preserve some geometry quantity, such as the area of the region bounded by the curve. For this, one may see 13 for a recent study.

The main result of this paper is the following theorem.

Theorem 1. Suppose $\gamma(u, 0)$ is a convex curve in the plane $R^{2}$. Assume $\gamma(t):=$ $\gamma(u, t)$ satisfies the following evolving equation

$$
\frac{\partial}{\partial t} \gamma(t)=(k-\alpha(t)) N,
$$

1991 Mathematics Subject Classification. 35K15, 35K55, 53A04.

Key words and phrases. non-local flow, length preserving, curve flow.

The research is partially supported by the National Natural Science Foundation of China 10631020 and SRFDP 20060003002. 
where $k$ is the curvature of the curve $\gamma(t)$ and

$$
\alpha(t)=\frac{1}{2 \pi} \int k^{2} d s
$$

Then the flow (1.1) is a length preserving flow. Furthermore, we have the global flow $\gamma(t)$ and $\gamma(t)$ converges in $C^{\infty}$ to the circle of of the fixed length $L$, as $t \rightarrow \infty$.

We remark that for $\alpha(t)=\frac{2 \pi}{L}$, where $L$ is the length of the curve $\gamma(t)$, the evolution equation (1.1) is area-preserving flow, which was studied by M.Gage in [6].

The paper is organized as follows.

In section 2, we introduce necessary formulae for the flow 1.1). We obtain key estimates about the curvature of the curve flow (1.1) in section 3 We can justify the assumption that the curve is a convex curve and the flow does not blow up in finite time. We obtain the theorem 1 in the $C^{0}$ case. In the last section, we show the $C^{\infty}$ convergence of the flow.

\section{Preparation}

First of all, we derive basic formulae for our curve flow (1.1).

Lemma 2. Let $w=\left|\gamma_{u}\right|$. Then we have

$$
w_{t}=-k(k-\alpha(t)) w
$$

and

$$
\frac{\partial}{\partial t} \frac{\partial}{\partial s}-\frac{\partial}{\partial s} \frac{\partial}{\partial t}=k(k-\alpha)
$$

Proof. Note that

$$
w^{2}=\left|\gamma_{u}\right|^{2}
$$

Then we have

$$
w w_{t}=<\gamma_{u}, \gamma_{t u}>=<\gamma_{u},((k-\alpha) N)_{u}>=w^{2}(k-\alpha)<T, N_{s}>.
$$

Using

$$
N_{s}=-k T,
$$

we have

$$
w_{t}=-k(k-\alpha) w
$$

So,

$$
\begin{aligned}
\frac{\partial}{\partial t} \frac{\partial}{\partial s}-\frac{\partial}{\partial s} \frac{\partial}{\partial t} & =\frac{\partial}{\partial t}\left(\frac{1}{w}\right) \frac{\partial}{\partial u} \\
& =k(k-\alpha) \partial_{s} .
\end{aligned}
$$

Recall that $d s=w d u$. Then we have

$$
(d s)_{t}=w_{t} d u=-k(k-\alpha) d s .
$$

We shall use this formula later.

\section{Lemma 3.}

$$
\frac{\partial}{\partial t} T=\partial_{s} k N
$$


Proof.

$$
\begin{aligned}
\frac{\partial}{\partial t} T & =\frac{\partial}{\partial t} \frac{\partial}{\partial s} \gamma=\frac{\partial}{\partial s} \frac{\partial}{\partial t} \gamma+k(k-\alpha) \partial_{s} \gamma \\
& =\partial_{s}((k-\alpha) N)+k(k-\alpha) T \\
& =\partial_{s} k N .
\end{aligned}
$$

We denote the angle between the tangent and the $\mathrm{X}$-axis by $\theta$. Then we have

$$
\cos \theta=<T, X>
$$

and

$$
k=\frac{\partial \theta}{\partial s} .
$$

Lemma 4.

$$
\frac{\partial \theta}{\partial t}=\partial_{s} k
$$

Proof.

$$
-\sin \theta \frac{\partial \theta}{\partial t}=<\frac{\partial T}{\partial t}, X>=\partial_{s} k<N, X>
$$

Then we can derive the important evolution equation for curvatures.

\section{Lemma 5 .}

$$
\frac{\partial}{\partial t} k=\partial_{s}^{2} k+k^{2}(k-\alpha)
$$

Proof.

$$
\frac{\partial}{\partial t} \frac{\partial}{\partial s} \theta=\frac{\partial}{\partial s} \frac{\partial}{\partial t} \theta+k(k-\alpha) \partial_{s} \theta=\partial_{s}^{2} k+k^{2}(k-\alpha) .
$$

Hence, we have

\section{Lemma 6.}

$$
\frac{\partial}{\partial t} \int k d s=0
$$

Proof.

$$
\frac{\partial}{\partial t} \int k d s=\int \frac{\partial}{\partial t} k d s+k \frac{\partial}{\partial t} d s=\int \partial_{s}^{2} k+k^{2}(k-\alpha)-k^{2}(k-\alpha) d s=0 .
$$

Using this, we now derive

Remark 7. Since $\gamma(0)$ is a convex curve in the plane, we have $\int k d s=2 \pi$.

Hence, we have

Lemma 8. The length of the curve is fixed under the flow. 
Proof.

$$
\begin{aligned}
\frac{\partial}{\partial t} L & =\int \frac{<\gamma_{u}, \gamma_{t u}>}{\left|\gamma_{u}\right|} d u=\int<T, \gamma_{t s}>d s \\
& =\int<T,((k-\alpha) N)_{s}>d s=-\int k(k-\alpha) d s \\
& =-\int k^{2} d s+\alpha \int k d s=2 \pi \alpha-\int k^{2} d s=0 .
\end{aligned}
$$

Another important fact for us is the following

Lemma 9. The area of the disk bounded by the curve $\gamma(t)$ is increasing. That is,

$$
\frac{d}{d t} A(t)=\alpha L-2 \pi \geq 0 .
$$

Proof. Since

$$
-2 A(t)=\int<\gamma, N>d s,
$$

we have

$$
\begin{aligned}
-2 \frac{d}{d t} A(t)= & \int \frac{d}{d t}<\gamma, N>d s+\int<\gamma, N>\frac{d}{d t} d s \\
= & \int<(k-\alpha) N, N>d s+\int<\gamma,-\partial_{s}(k-\alpha) T>d s \\
& +\int<\gamma, N>(-k(k-\alpha)) d s \\
= & \int(k-\alpha) d s-\int \partial_{s}(k-\alpha)<\gamma, T>d s-\int<\gamma, N>k(k-\alpha) d s \\
= & \int(k-\alpha) d s+\int(k-\alpha)(<T, T>+k<\gamma, N>) d s \\
& -\int<\gamma, N>k(k-\alpha) d s \\
= & 2 \int(k-\alpha) d s=2(2 \pi-L \alpha)
\end{aligned}
$$

Since

$$
2 \pi \alpha=\int k^{2} d s \geq \frac{1}{L}\left(\int k d s\right)^{2}=\frac{4 \pi^{2}}{L} .
$$

So

$$
2 \pi-\alpha L \leq 0,
$$

which implies the result wanted.

We remark that the above formula takes the equality when $k$ is a constant, i.e. the curve is a circle.

We now consider the growth of the support function

$$
P:=<X, N>
$$

and the growth of $\alpha$. 
Lemma 10.

$$
\begin{gathered}
\partial_{t} P=\partial_{s}^{2} P+2 k-\alpha+k^{2} P . \\
\partial_{t} \alpha=-\frac{1}{\pi} \int\left(k_{s}\right)^{2} d s+\frac{1}{2 \pi} \int k^{3}(k-\alpha) d s . \\
\partial_{t}\left(\frac{P}{k}\right)=\partial_{s}^{2}\left(\frac{P}{k}\right)+\left(P-\frac{1}{k}\right) \alpha+2 \frac{k_{s}}{k} \partial_{s}\left(\frac{P}{k}\right) .
\end{gathered}
$$

Proof.

$$
\begin{gathered}
\partial_{t} P=\partial_{t}<\gamma, N>=<\partial_{t} \gamma, N>+<\gamma, \partial_{t} N> \\
=k-\alpha-k_{s}<\gamma, T>. \\
\partial_{s} P=\partial_{s}<\gamma, N>=-k<\gamma, T>. \\
\partial_{s}^{2} P=-k_{s}<\gamma, T>-k-k^{2}<\gamma, N>.
\end{gathered}
$$

Combine the above computation, we have

$$
\begin{gathered}
\partial_{t} P=\partial_{s}^{2} P+2 k-\alpha+k^{2} P . \\
\partial_{t}\left(\frac{k}{P}\right)=\frac{k_{t} P-k P_{t}}{P^{2}} . \\
\partial s\left(\frac{k}{p}\right)=\frac{k_{s} P-k P_{s}}{P^{2}} . \\
\partial_{s}^{2}\left(\frac{k}{P}\right)=\frac{k_{s s} P-k P_{s s}}{P^{2}}-2 \frac{P_{s}}{P} \partial_{s}\left(\frac{k}{P}\right) .
\end{gathered}
$$

Combine the above computation, we have

$$
\begin{aligned}
\partial_{t}\left(\frac{k}{P}\right)-\partial_{s}^{2}\left(\frac{k}{P}\right) & =\frac{\left(k_{t}-k_{s s}\right) P-k\left(P_{t}-P_{s s}\right)}{P^{2}}+2 \frac{P_{s}}{P} \partial_{s}\left(\frac{k}{P}\right) \\
& =\frac{k^{2}(k-\alpha) P-k\left(2 k-\alpha+k^{2} P\right)}{P^{2}}+2 \frac{P_{s}}{P} \partial_{s}\left(\frac{k}{P}\right) \\
& =\frac{\alpha(1-k P)-2 k^{2}}{P^{2}}+2 \frac{P_{s}}{P} \partial_{s}\left(\frac{k}{P}\right) . \\
\partial_{t} \alpha=\frac{1}{\pi} \int k k_{t} d s-\frac{1}{2 \pi} \int k^{3}(k-\alpha) d s & \frac{1}{\pi} \int k\left(k_{s s}+k^{2}(k-\alpha)\right) d s-\frac{1}{2 \pi} \int k^{3}(k-\alpha) d s \\
= & -\frac{1}{\pi} \int\left(k_{s}\right)^{2} d s+\frac{1}{2 \pi} \int k^{3}(k-\alpha) d s .
\end{aligned}
$$

In principle we may consider the evolution of $f:=\frac{k}{P-\rho}$ for $\rho$ is a circle inside the region bounded by $\gamma(t)$ and get control of the curvature of the evolving curve. However, unlike the shrinking flow where $\alpha=0$, we can not use the maximum principle to get the bound of $f$ to show the convergence of the flow. So we need to use entropy estimate and integral estimate as done by Gage and Hamilton for the shrinking curve flow [7. These will be done in below. 


\section{LONG TIME EXISTENCE}

In this section, we derive key estimates of the curvature $k$ of the evolving curve $\gamma(t)$.

In the following, we work with the general curve flow

$$
\frac{\partial}{\partial t} \gamma=(k-\alpha) N+\eta T
$$

where $\eta$ will be determined later.

Similar to above, we have basic formulae

\section{Lemma 11.}

$$
\begin{gathered}
\partial_{t} \partial_{s}-\partial_{s} \partial_{t}=k(k-\alpha) \partial_{s}-\eta_{s} \partial_{s} . \\
\partial_{t} T=\left(\partial_{s}(k-\alpha)+k \eta\right) N . \\
\partial_{t} \theta=\partial_{s} k+k \eta . \\
\partial_{t} L=0 . \\
\partial_{t} A=\int(\alpha-k) d s .
\end{gathered}
$$

We take $\eta$ such that $\partial_{t} \theta=0$, i.e. $\eta=-\partial_{\theta} k$.

Remark 12. Under this flow, the length is also preserved, and the variation of area is the same as $\eta=0$.

Lemma 13.

$$
\frac{\partial k}{\partial t}=k^{2} \partial_{\theta}^{2} k+k^{2}(k-\alpha)
$$

Proof.

$$
\begin{aligned}
\partial_{t} k & =\partial_{t} \partial_{s} \theta=\partial_{s} \partial_{t} \theta+k(k-\alpha) \partial_{s} \theta-\partial_{s} \eta k \\
& =k^{2} \partial_{\theta}^{2} k+k^{2}(k-\alpha) .
\end{aligned}
$$

Theorem 14. Convexity is preserved along the flow (3.1). In fact, for any finite time $T<\infty$, such that the curve flow exists on $[0, T]$, we have $k(t)$ is uniformly bounded from below on $t \in[0, T]$

Proof. By direct computation, we have

$$
\partial_{t}\left(\frac{1}{k}-\frac{A}{L}-\frac{2 \pi t}{L}\right)=k^{2} \partial_{\theta}^{2}\left(\frac{1}{k}-\frac{A}{L}-\frac{2 \pi t}{L}\right)-2 k^{3}\left(\partial_{\theta}\left(\frac{1}{k}-\frac{A}{L}-\frac{2 \pi t}{L}\right)\right)^{2}-k .
$$

Before $\frac{1}{k}-\frac{A}{L}-\frac{2 \pi t}{L}$ blow up, we have $k>0$.

Since the curve is compact, we can take the maximum of $\frac{1}{k}-\frac{A}{L}-\frac{2 \pi t}{L}$ at $\left(x_{0}, t_{0}\right) \in$ $\gamma \times[0, T]$, where the curve flow exists on $[0, T]$. If $t_{0}>0$, we have

$$
\left.\frac{\partial}{\partial t}\left(\frac{1}{k}-\frac{A}{L}-\frac{2 \pi t}{L}\right)\right|_{\left(x_{0}, t_{0}\right)} \geq 0 \text {. }
$$

But

$$
\left.k^{2} \partial_{\theta}^{2}\left(\frac{1}{k}-\frac{A}{L}-\frac{2 \pi t}{L}\right)\right|_{x_{0}, t_{0}} \leq 0,\left.2 k^{3}\left(\partial_{\theta}\left(\frac{1}{k}-\frac{A}{L}-\frac{2 \pi t}{L}\right)\right)^{2}\right|_{x_{0}, t_{0}}=0,-k<0
$$


A contradiction. So

$$
\frac{1}{k}-\frac{A}{L}-\frac{2 \pi t}{L} \leq \max \left(\frac{1}{k(0)}\right)-\frac{A(0)}{L}
$$

That is $k(t) \geq \frac{1}{C_{1}+C_{2} t}=c(t)$, where $C_{1}$ and $C_{2}$ are positive constant. So the convexity is preserved along the flow.

Theorem 15. $\int \log k(\theta, t) d \theta$ is non increasing along the flow, So there is a uniform bound of $\int \log k(\theta, t) d \theta$ along the flow.

Proof.

$$
\begin{aligned}
\frac{\partial}{\partial t} \int \log k(\theta, t) d \theta & =\int k \partial_{\theta}^{2} k+k(k-\alpha) d \theta \\
& =\int_{0}^{2 \pi}-\left(\partial_{\theta} k\right)^{2}+(k-\alpha)^{2} d \theta+\alpha \int_{0}^{2 \pi}(k-\alpha) d \theta .
\end{aligned}
$$
have

By definition, we have $\int_{0}^{2 \pi}(k-\alpha) d \theta=0$. Using the Wirtinger inequality, we

$$
\frac{\partial}{\partial t} \int_{0}^{2 \pi} \log k(\theta, t) d \theta \leq 0
$$

Hence,

$$
\int_{0}^{2 \pi} \log k(\theta, t) d \theta \leq \int_{0}^{2 \pi} \log k(\theta, 0) d \theta
$$

Theorem 16. 7] Suppose the curve flow exists on $[0, T)$. For any $\delta>0$, we can find a constant $C(T)$ such that $k(\theta, t) \leq C(T)$ except on intervals of length less than or equal to $\delta$.

Proof. If $k \geq C(t)$ on $a \leq \theta \leq b$ and $b-a \geq \delta$, then

$$
\begin{aligned}
\int_{0}^{2 \pi} \log k(\theta, t) d \theta & \geq \delta \log C(T)+(2 \pi-\delta) \log k_{m i n}(t) \\
& \geq \delta \log C(T)+(2 \pi-\delta) \log c(T)
\end{aligned}
$$

where $c(T)$ is the low bound of $k(t)$ on $[0, T)$. Since $\int_{0}^{2 \pi} \log k(\theta, t) d \theta$ is non increasing, $C(T)$ is bounded above.

Lemma 17. We have

$$
\int\left(\frac{\partial k}{\partial \theta}\right)^{2} d \theta \leq \int k^{2}+D
$$

where $D$ is a constant which depends only on $\gamma(0)$.

Proof.

$$
\begin{aligned}
& \frac{\partial}{\partial t} \int(k-\alpha)^{2}-\left(\frac{\partial k}{\partial \theta}\right)^{2} d \theta \\
= & 2 \int(k-\alpha)\left(\partial_{t} k-\partial_{t} \alpha\right)-2 \frac{\partial k}{\partial \theta} \frac{\partial^{2} k}{\partial \theta \partial t} d \theta \\
= & 2 \int\left(k-\alpha+\partial_{\theta}^{2} k\right) \partial_{t} k d \theta-2 \int(k-\alpha) \partial_{t} \alpha d \theta \\
= & 2 \int\left(k-\alpha+\partial_{\theta}^{2} k\right)^{2} k^{2} d \theta+2 \partial_{t} \alpha\left(2 \pi \alpha-\int k d \theta\right)
\end{aligned}
$$


But

$$
\int k d \theta=\int k^{2} d s=2 \pi \alpha .
$$

So

$$
\frac{\partial}{\partial t} \int(k-\alpha)^{2}-\left(\frac{\partial k}{\partial \theta}\right)^{2} d \theta=2 \int\left(k-\alpha+\partial_{\theta}^{2} k\right)^{2} k^{2} d \theta
$$

Integrating the above inequality, we have

$$
\int(k(t)-\alpha(t))^{2}-\left(\frac{\partial k(t)}{\partial \theta}\right)^{2} d \theta \geq \int(k(0)-\alpha(0))^{2}-\left(\frac{\partial k(0)}{\partial \theta}\right)^{2} d \theta=-D .
$$

Then we have

$$
\int\left(\frac{\partial k(t)^{2}}{\partial \theta}\right) d \theta \leq \int(k(t)-\alpha(t))^{2}+D \leq \int k^{2} d \theta+D .
$$

Theorem 18. If $\int_{0}^{2 \pi} \log k(\theta, t) d \theta$ is bounded on $[0, T)$, then $k(\theta, t)$ is uniformly bounded on $\gamma \times[0, T)$.

Proof. For any given $\delta$, by the above estimate, we have $k \leq C(T)$ except on intervals $[a, b]$ of length less than $\delta$. On such an interval

$k(\phi)=k(a)+\int_{a}^{\phi} \frac{\partial k}{\partial \theta} d \theta \leq C(T)+\sqrt{\delta}\left(\int\left(\frac{\partial k}{\partial \theta}\right)^{2} d \theta\right)^{1 / 2} \leq C(T)+\sqrt{\delta}\left(\int k^{2} d \theta+D\right)^{1 / 2}$.

This shows that if $k_{\max }$ is the maximum value of $k$, then

$$
k_{\max } \leq C(T)+\sqrt{\delta}\left(2 \pi k_{\max }^{2}+D\right)^{1 / 2} .
$$

By choosing $\delta$ small, we have

$$
k_{\max }^{2} \leq \frac{2 C^{2}(T)+2 \delta D}{1-4 \pi \delta} \leq 4 C^{2}(T) .
$$

Lemma 19. If $k$ is bounded, then $\frac{\partial k}{\partial \theta}$ is bounded.

Proof.

$$
\partial_{t} \partial_{\theta} k=k^{2} \partial_{\theta}^{3} k+2 k \partial_{\theta} k \partial_{\theta}^{2} k+3 k^{2} \partial_{\theta} k-2 \alpha k \partial_{\theta} k .
$$

Since $k$ is bounded, $\alpha$ is bounded. So $\partial_{\theta} k$ grows at most exponentially.

In the following, we will use $k^{\prime}$, etc, to denote the derivatives of $\gamma(t)$ w.r.t variable $\theta, \partial_{\theta} k$, etc.

Lemma 20. If $k$ and $k^{\prime}$ are bounded, then $\int_{0}^{2 \pi}\left(k^{\prime \prime}\right)^{4} d \theta$ is bounded. 
Proof.

$$
\begin{aligned}
\frac{\partial}{\partial t} \int_{0}^{2 \pi}\left(k^{\prime \prime}\right)^{4} d \theta= & 4 \int_{0}^{2 \pi}\left(k^{\prime \prime}\right)^{3}\left(k^{2} k^{\prime \prime}+k^{2}(k-\alpha)\right)^{\prime \prime} d \theta \\
= & -12 \int_{0}^{2 \pi}\left(k^{\prime \prime}\right)^{2}\left(k^{\prime \prime \prime}\right)\left(k^{2} k^{\prime \prime \prime}+2 k k^{\prime} k^{\prime \prime}+3 k^{2} k^{\prime}-2 \alpha k k^{\prime}\right) d \theta \\
= & -12 \int_{0}^{2 \pi} k^{2}\left(k^{\prime \prime}\right)^{2}\left(k^{\prime \prime \prime}\right)^{2}+2 k k^{\prime}\left(k^{\prime \prime}\right)^{3} k^{\prime \prime \prime}+3 k^{2} k^{\prime}\left(k^{\prime \prime}\right)^{2} k^{\prime \prime \prime} d \theta \\
& +24 \alpha \int_{0}^{2 \pi} k k^{\prime}\left(k^{\prime \prime}\right)^{2} k^{\prime \prime \prime} d \theta \\
\leq & C_{1} \int_{0}^{2 \pi}\left(k^{\prime \prime}\right)^{4}\left(k^{\prime}\right)^{2} d \theta+C_{2} \int_{0}^{2 \pi} k^{2}\left(k^{\prime}\right)^{2}\left(k^{\prime \prime}\right)^{2} d \theta \\
& +24 \alpha C_{3} \int_{0}^{2 \pi}\left(k^{\prime}\right)^{2}\left(k^{\prime \prime}\right)^{2} d \theta
\end{aligned}
$$

By the bound of $k, k^{\prime}$, we see that $\int_{0}^{2 \pi}\left(k^{\prime \prime}\right)^{4}$ grows at most exponentially.

Lemma 21. If $k, k^{\prime}$ and $\int_{0}^{2 \pi}\left(k^{\prime \prime}\right)^{4} d \theta$ are bounded, so is $\int_{0}^{2 \pi}\left(k^{\prime \prime \prime}\right)^{2} d \theta$.

Proof.

$$
\begin{aligned}
& \frac{\partial}{\partial \theta} \int_{0}^{2 \pi}\left(k^{\prime \prime \prime}\right)^{2} d \theta \\
= & -2 \int_{0}^{2 \pi} k^{\prime \prime \prime \prime}\left(k^{2} k^{\prime \prime}+k^{3}-\alpha k^{2}\right)^{\prime \prime} d \theta \\
= & -2 \int_{0}^{2 \pi} k^{2}\left(k^{\prime \prime \prime \prime}\right)^{2}+4 k k^{\prime} k^{\prime \prime \prime} k^{\prime \prime \prime \prime}+2 k\left(k^{\prime \prime}\right)^{2} k^{\prime \prime \prime \prime} \\
& +2\left(k^{\prime}\right)^{2} k^{\prime \prime} k^{\prime \prime \prime \prime}+3 k^{2} k^{\prime \prime} k^{\prime \prime \prime \prime}+6 k\left(k^{\prime}\right)^{2} k^{\prime \prime \prime \prime} \\
& -2 \alpha\left(k^{\prime}\right)^{2} k^{\prime \prime \prime \prime}-2 \alpha k k^{\prime \prime} k^{\prime \prime \prime \prime} d \theta \\
\leq & C_{1} \int\left(k^{\prime}\right)^{2}\left(k^{\prime \prime \prime}\right)^{2} d \theta+C_{2} \int\left(k^{\prime \prime}\right)^{4} d \theta+C_{3} \int \frac{\left(k^{\prime}\right)^{4}}{k^{2}}\left(k^{\prime \prime}\right)^{2} d \theta \\
+ & C_{4} \int k^{2}\left(k^{\prime \prime}\right)^{2} d \theta+C_{5} \int\left(k^{\prime}\right)^{4} d \theta+C_{6} \int \frac{\left(k^{\prime}\right)^{4}}{k^{2}} d \theta+C_{7} \int\left(k^{\prime \prime}\right)^{2} d \theta .
\end{aligned}
$$

By the bounds of $k, k^{\prime},\left\|k^{\prime \prime}\right\|_{4}$, we see that $\int_{0}^{2 \pi}\left(k^{\prime \prime \prime}\right)^{2} d \theta$ grows at most exponentially.

Corollary 22. Under the same hypothesis, $k^{\prime \prime}$ is bounded.

Proof. In one dimension

$$
\max |f|^{2} \leq C \int\left|f^{\prime}\right|^{2}+f^{2}
$$

We apply this to $k^{\prime \prime}$.

Lemma 23. If $k, k^{\prime}$, and $k^{\prime \prime}$ are uniformly bounded, then so are $k^{\prime \prime \prime}$ and all the higher derivatives. 
Proof. We compute

$$
\begin{aligned}
\frac{\partial}{\partial t} k^{\prime \prime \prime}= & \left(k^{2} k^{\prime \prime}+k^{3}-k^{2} \alpha\right)^{\prime \prime \prime} \\
= & k^{2} k^{v}+6 k k^{\prime} k^{i v}+\left(8 k k^{\prime \prime}+6 k^{\prime 2}+3 k^{2}-2 \alpha k\right) k^{\prime \prime \prime} \\
& +\left(6 k^{\prime}\left(k^{\prime \prime}\right)^{2}+18 k k^{\prime} k^{\prime \prime}+6\left(k^{\prime}\right)^{3}-6 \alpha k^{\prime} k^{\prime \prime}\right)
\end{aligned}
$$

Since $k, k^{\prime}, k^{\prime \prime}, \alpha$ is bounded, $k^{\prime \prime \prime}$ grows at most exponentially. Similarly, we can show that $k^{(n)}$ is bounded on finite intervals.

From the above analysis, we have

Theorem 24. The curve flow does not blow up in finite time.

Theorem 25. 5] For the closed, convex $C^{2}$ curve $\gamma(t)$ in the plane, we have

$$
\pi \frac{L}{A} \leq \int_{0}^{L} k^{2} d s
$$

where $L, A$ and $k$ are the length of the curve, the area it encloses, and its curvature respectively.

Theorem 26. If the convex curve $\gamma(t)$ evolves according to (3.1), then the isoperimetric deficit $L^{2}-4 \pi A$ is decreasing during the evolution process (1.1) and converges to zero as the time $t$ goes to infinity.

Proof. Since the length of the curve is preserved,

$$
\frac{d}{d t}\left(L^{2}-4 \pi A\right)=-4 \pi \frac{d}{d t} A(t)=-4 \pi(L \alpha-2 \pi) \leq 0 .
$$

From the above theorem 25, we have

$$
\frac{d}{d t}\left(L^{2}-4 \pi A\right) \leq-4 \pi\left(\frac{L^{2}}{2 A}-2 \pi\right)=-\frac{2 \pi}{A}\left(L^{2}-4 \pi A\right),
$$

Since for any plane curve,

$$
\frac{L^{2}}{4 \pi} \geq A,
$$

we have

$$
\frac{d}{d t}\left(L^{2}-4 \pi A\right) \leq-\frac{8 \pi^{2}}{L^{2}}\left(L^{2}-4 \pi A\right)
$$

So

$$
L^{2}-4 \pi A(t) \leq C \exp \left(-\frac{8 \pi^{2}}{L^{2}} t\right)
$$

As $t \rightarrow \infty$, we have

$$
L^{2}-4 \pi A \rightarrow 0 .
$$

By Bonnesen inequality (see [14]), $\frac{L^{2}}{A}-4 \pi \geq \frac{\pi^{2}}{A}\left(r_{\text {out }}-r_{\text {in }}\right)^{2}$, we have $r_{\text {out }}-r_{\text {in }} \rightarrow$ 0 , as $t \rightarrow \infty$. So the curve converge to a circle in Hausdorff sense. 


\section{4. $C^{\infty}$ CONVERGENCE}

We will follow Hamilton's estimate to prove the $C^{\infty}$ convergence.

Define

$$
k_{w}^{*}=\sup \{b \mid k(\theta)>b \text { on some interval of length } w\} .
$$

Lemma 27. 7

$$
k_{w}^{*}(t) r_{i n}(t) \leq \frac{1}{1-K(w)\left(\frac{r_{o u t}}{r_{\text {in }}}-1\right)},
$$

where $r_{\text {in }}$ and $r_{\text {out }}$ are the radii of the largest inscribed circle and the smallest circumscribed circle of the curve defined by the $k(;, t)$ respectively, and $K$ is a positive decreasing function of $w$ with $K(0)=\infty$ and $K(\pi)=0$.

Remark 28. Here we need the explicit formula of $K(w)$.

$$
K(w)=\frac{2 \cos \left(\frac{w}{2}\right)}{1-\cos \left(\frac{w}{2}\right)} .
$$

See also [7.

By the above section, we have the curve $\gamma(t)$ converges to a circle in Hausdorff sense as $t \rightarrow \infty$, i.e.

$$
\pi\left(r_{\text {out }}-r_{\text {in }}\right)^{2} \leq L^{2}-4 \pi A \rightarrow 0 .
$$

We also have $\pi r_{\text {out }}^{2} \geq A(t) \geq A(0)$, and $r_{\text {out }} \rightarrow r_{\text {in }}$, So There is a sufficient large time $T_{1}$ such that $r_{i n}(t) \geq \sqrt{\frac{A_{0}}{2 \pi}}$ for $t \geq T_{1}$.

We first fix a small $w$. Then there is a sufficient large time $T_{2} \geq T_{1}$, such that

$$
K(w)\left(\frac{r_{\text {out }}}{r_{\text {in }}}-1\right) \leq 1 / 2 .
$$

for $t \geq T_{2}$. By the theorem 27, we have $k_{w}^{*}(t) r_{i n}(t) \leq 2$, i.e $k_{w}^{*}(t) \leq 2 \sqrt{\frac{2 \pi}{A_{0}}}$ for $t \geq T_{2}$.

Then by a use of the method used by Gage and Hamilton in [7, we have

Theorem 29. Curvature $k(t)$ uniformly bounded along the flow.

Proof. We just need to consider the curvature $k$ of the curve $\gamma(t)$ for $t \geq T_{2}$. First we fix a small $w<\left(\frac{1}{4 \pi}\right)^{2}$. Suppose that $[a, b]$ is a interval such that $k \geq k_{w}^{*}$. By condition, we have $|b-a| \leq w$ and $k(a)=k_{w}^{*}$. For any $\phi \in[a, b]$, we have

$$
\begin{gathered}
k(\phi)=\quad k(a)+\int_{a}^{\phi} \frac{\partial k}{\partial \theta} d \theta \leq k_{w}^{*}+\sqrt{w}\left(\int\left(\frac{\partial k}{\partial \theta}\right)^{2} d \theta\right)^{1 / 2} \\
\leq k_{w}^{*}+\sqrt{w}\left(\int k^{2} d \theta+D\right)^{1 / 2} .
\end{gathered}
$$

If $k_{\max }$ is the maximum value of $k$, then

$$
k_{\max } \leq k_{w}^{*}+\sqrt{w}\left(2 \pi k_{\max }^{2}+D\right)^{1 / 2} \leq k_{w}^{*}+2 \pi \sqrt{w} k_{\max }+\sqrt{w} D .
$$

So for $t \geq T_{2}$, we have $k$ is bounded uniformly.

Since $k(\theta, t)$ is uniformly bounded, $\int\left(\partial_{\theta} k\right)^{2} d \theta$ is also uniformly bounded. Then we have $k(\theta, t)$ is equi-continuous. So for any sequence $k\left(\theta, t_{i}\right)$, we can choose a sequence $k\left(\theta, t_{i_{n}}\right)$ converge uniformly to $k(\theta, \infty)$. But we know the curve converge to circle in the Hausdorff sense. So $k(\theta, \infty)=$ const. Since every subsequences converge to $k(\theta, \infty)=$ const, we have $k(\theta, t)$ converge to $k(\theta, \infty)=$ const uniformly.

Similar to [7, we have 
Lemma 30. $\left\|k^{\prime}\right\|_{4}$ are bounded by constants independent of $t$.

Proof.

$$
\begin{aligned}
\frac{\partial}{\partial t} \int\left(k^{\prime}\right)^{4} d \theta= & 4 \int\left(k^{\prime}\right)^{3}\left(k^{2} k^{\prime \prime}+k^{2}(k-\alpha)\right)^{\prime} d \theta \\
= & -12 \int_{0}^{2 \pi} k^{2}\left(k^{\prime}\right)^{2}\left(k^{\prime \prime}\right)^{2}-12 \int_{0}^{2 \pi}\left(k^{\prime}\right)^{2} k^{\prime \prime} k^{3} d \theta \\
& -8 \alpha \int_{0}^{2 \pi}\left(k^{\prime}\right)^{4} k d \theta \\
\leq & 3 \int_{0}^{2 \pi} k^{4}\left(k^{\prime}\right)^{2} d \theta-8 \alpha \int_{0}^{2 \pi}\left(k^{\prime}\right)^{4} d \theta
\end{aligned}
$$

Since $k$ converges to a constant, $\alpha(t)$ is converging to a constant. Using the Holder inequality, we have

$$
\frac{\partial f}{\partial t} \leq C_{1} f^{1 / 2}-C_{2} f
$$

where $f=\int_{0}^{2 \pi}\left(k^{\prime}\right)^{4} d \theta, C_{1}$ and $C_{2}$ is independent of $t$. By the lemma 5.7.4 of [7], we have $\left\|k^{\prime}\right\|_{4}(t)$ is uniformly bounded.

Lemma 31. $\left\|k^{\prime \prime}\right\|_{2}$ is bounded by a constant which is independent of $t$.

Proof.

$$
\begin{aligned}
& \partial_{t} \int_{0}^{2 \pi}\left(k^{\prime \prime}\right)^{2} d \theta \\
= & \int_{0}^{2 \pi} k^{\prime \prime}\left(\partial_{t} k\right)^{\prime \prime} d \theta \\
= & -\int_{0}^{2 \pi} k^{\prime \prime \prime}\left(k^{2} k^{\prime \prime}\right)^{\prime} d \theta+\int_{0}^{2 \pi} k^{\prime \prime}\left(\left(k^{3}\right)^{\prime \prime}-\alpha\left(k^{2}\right)^{\prime \prime}\right) d \theta \\
= & -\int_{0}^{2 \pi} k^{2}\left(k^{\prime \prime \prime}\right)^{2} d \theta-2 \int_{0}^{2 \pi} k k^{\prime} k^{\prime \prime} k^{\prime \prime \prime} d \theta \\
& -3 \int_{0}^{2 \pi} k^{2} k^{\prime} k^{\prime \prime \prime} d \theta-2 \alpha \int_{0}^{2 \pi} k\left(k^{\prime \prime}\right)^{2} d \theta-2 \alpha \int_{0}^{2 \pi} k^{\prime \prime}\left(k^{\prime}\right)^{2} d \theta
\end{aligned}
$$

By the Cauchy inequality, we have

$$
\begin{aligned}
\partial_{t} \int_{0}^{2 \pi}\left(k^{\prime \prime}\right)^{2} d \theta \leq & C_{1} \int_{0}^{2 \pi}\left(k^{\prime} k^{\prime \prime}\right)^{2} d \theta+C_{2} \int_{0}^{2 \pi} k^{2}\left(k^{\prime}\right)^{2} d \theta-2 \alpha \int_{0}^{2 \pi} k\left(k^{\prime \prime}\right)^{2} d \theta \\
& -2 \alpha \int_{0}^{2 \pi} k^{\prime \prime}\left(k^{\prime}\right)^{2} d \theta
\end{aligned}
$$


We can control the first term by the inequality in above lemma 30.

$$
\begin{aligned}
\partial_{t} \int_{0}^{2 \pi}\left(k^{\prime \prime}\right)^{2} d \theta \leq & C_{2} \int_{0}^{2 \pi} k^{2}\left(k^{\prime}\right)^{2} d \theta-2 \alpha \int_{0}^{2 \pi} k\left(k^{\prime \prime}\right)^{2} d \theta \\
& -2 \alpha \int_{0}^{2 \pi} k^{\prime \prime}\left(k^{\prime}\right)^{2} d \theta-C_{3} \partial_{t} \int_{0}^{2 \pi}\left(k^{\prime}\right)^{4} d \theta \\
& -C_{4} \int_{0}^{2 \pi}\left(k^{\prime}\right)^{2} k^{\prime \prime} k^{3} d \theta-C_{5} \int_{0}^{2 \pi}\left(k^{\prime}\right)^{4} d \theta \\
& \leq C_{6}-C_{7} \int_{0}^{2 \pi}\left(k^{\prime \prime}\right)^{2} d \theta-C_{3} \partial_{t} \int_{0}^{2 \pi}\left(k^{\prime}\right)^{4} d \theta
\end{aligned}
$$

We denote $\int_{0}^{2 \pi}\left(k^{\prime \prime}\right)^{2} d \theta$ by $f(t)$. Then we have

$$
\partial_{t} f \leq C_{6}-C_{7} f-C_{3} \partial_{t} \int_{0}^{2 \pi}\left(k^{\prime}\right)^{4} d \theta
$$

Multiplying $e^{c_{7} t}$ on both side and integrating, we have

$$
\left.e^{c_{7} t} f(t)\right|_{0} ^{t} \leq C_{6} \int_{0}^{t} e^{C_{7} t} d t-C_{3} \int_{0}^{t} e^{C_{7} t} \partial_{t} \int_{0}^{2 \pi}\left(k^{\prime}\right)^{4} d \theta d t
$$

So we have

$$
\begin{aligned}
e^{C_{7} t} f(t) & \leq \frac{C_{6}}{C_{7}} e^{C_{7} t}+C_{3} C_{7} \int_{0}^{t} e^{C_{7} t} \int_{0}^{2 \pi}\left(k^{\prime}\right)^{4} d \theta+C_{8} \\
& \leq \frac{C_{6}}{C_{7}} e^{C_{7} t}+C_{3} M e^{C_{7} t}+C_{8}
\end{aligned}
$$

where $M$ is the bound of $\int_{0}^{2 \pi}\left(k^{\prime}\right)^{4} d \theta$ which is independent of $t$. So $\int_{0}^{2 \pi}\left(k^{\prime \prime}\right)^{2} d \theta$ is uniformly bounded.

As to lemma 5.7 .8 of $[7$, we have

Lemma 32. $\left\|k^{\prime}\right\|_{\infty}$ converges to 0 as $t \rightarrow \infty$.

Lemma 33. 7] For any $0<\beta<1$ we can choose $A$ so that for $t>A$

$$
\int\left(k^{\prime \prime}\right)^{2} d \theta \geq 4 \beta \int\left(k^{\prime}\right)^{2} d \theta .
$$

Lemma 34. For any $0<\beta<1$, there is a constant $C_{1}$ such that $\left\|k^{\prime}\right\|_{2} \leq$ $C_{1} e^{-2 \beta C^{2} t}$, where $k \rightarrow C$, as $t \rightarrow \infty$.

Proof.

$$
\begin{aligned}
\partial_{t} \int\left(k^{\prime}\right)^{2} d \theta & =2 \int k^{\prime}\left(k^{2} k^{\prime \prime}+k^{3}-\alpha k^{2}\right)^{\prime} d \theta \\
& =-2 \int k^{2}\left(k^{\prime \prime}\right)^{2} d \theta+6 \int k^{2}\left(k^{\prime}\right)^{2} d \theta-4 \alpha \int k\left(k^{\prime}\right)^{2} d \theta
\end{aligned}
$$

Since $k \rightarrow C$, as $t \rightarrow \infty$, we have

$$
\partial_{t} \int\left(k^{\prime}\right)^{2} d \theta \leq-2 \beta C^{2} \int\left(k^{\prime}\right)^{2} d \theta
$$

So we complete the lemma. 
Lemma 35. We can find a constant $C$ such that $\left\|k^{\prime \prime}\right\|_{2} \leq C e^{-2 \beta t}$, where $\beta>0$ is a uniform constant.

Proof. By direct computation, we have

$$
\begin{aligned}
& \partial_{t} \int\left(k^{\prime \prime}\right)^{2} d \theta \\
= & 2 \int k^{\prime \prime}\left(k_{t}\right)^{\prime \prime} d \theta=2 \int k^{\prime \prime}\left(k^{2} k^{\prime \prime}+k^{2}(k-\alpha)\right)^{\prime \prime} d \theta \\
= & -2 \int k^{2}\left(k^{\prime \prime \prime}\right)^{2} d \theta-4 \int k k^{\prime} k^{\prime \prime} k^{\prime \prime \prime} d \theta-6 \int k^{2} k^{\prime} k^{\prime \prime \prime} d \theta \\
& -4 \alpha \int\left(k^{\prime}\right)^{2} k^{\prime \prime}-4 \alpha \int k\left(k^{\prime \prime}\right)^{2} d \theta \\
\leq & -2 \int k^{2}\left(k^{\prime \prime \prime}\right)^{2} d \theta+4 \epsilon \int k^{2}\left(k^{\prime \prime \prime}\right)^{2} d \theta+1 / \epsilon \int\left(k^{\prime}\right)^{2}\left(k^{\prime \prime}\right)^{2} d \theta \\
& +6\left(\epsilon \int\left(k k^{\prime \prime \prime}\right)^{2}+1 / 4 \epsilon \int k^{2}\left(k^{\prime}\right)^{2}\right)-4 \alpha \int\left(k^{\prime}\right)^{2} k^{\prime \prime} d \theta \\
& -4 \alpha \int k\left(k^{\prime \prime}\right)^{2} d \theta
\end{aligned}
$$

First we choose $\epsilon>0$ small such that

$$
\begin{aligned}
\partial_{t} \int\left(k^{\prime \prime}\right)^{2} d \theta \leq & C_{1} \int\left(k^{\prime}\right)^{2}\left(k^{\prime \prime}\right)^{2} d \theta+C_{2} \int k^{2}\left(k^{\prime}\right)^{2} d \theta \\
& -4 \alpha \int\left(k^{\prime}\right)^{2} k^{\prime \prime} d \theta-4 \alpha \int k\left(k^{\prime \prime}\right)^{2} d \theta
\end{aligned}
$$

Since $\left\|k^{\prime}\right\|_{\infty}$ converges to $0, k \rightarrow C, \alpha \rightarrow C$ as $t \rightarrow \infty$, we have

$$
\partial_{t} \int\left(k^{\prime \prime}\right)^{2} d \theta \leq-2 C^{2} \int\left(k^{\prime \prime}\right)^{2} d \theta+C_{2} C e^{-C_{3} t}
$$

We denote $\int\left(k^{\prime \prime}\right)^{2} d \theta$ by $f$. Then we have

$$
\partial_{t} f \leq-2 C^{2} f+C_{2} C e^{-C_{3} t}
$$

By the lemma 5.7.6 of [7], we complete the proof.

Lemma 36. We can find uniform constants $C$ and $\beta>0$ such that $\left\|k^{\prime \prime}\right\|_{4} \leq C e^{-\beta t}$. 
Proof.

$$
\begin{aligned}
& \partial_{t} \int\left(k^{\prime \prime}\right)^{4} d \theta \\
= & 4 \int\left(k^{\prime \prime}\right)^{3}\left(k^{2} k^{\prime \prime}+k^{2}(k-\alpha)\right)^{\prime \prime} d \theta \\
= & -12 \int k^{2}\left(k^{\prime \prime}\right)^{2}\left(k^{\prime \prime \prime}\right)^{2} d \theta-24 \int k k^{\prime}\left(k^{\prime \prime}\right)^{3} k^{\prime \prime \prime} d \theta \\
& -36 \int k^{2} k^{\prime}\left(k^{\prime \prime}\right)^{2} k^{\prime \prime \prime} d \theta-8 \alpha \int\left(k^{\prime}\right)^{2}\left(k^{\prime \prime}\right)^{3} d \theta-8 \alpha \int k\left(k^{\prime \prime}\right)^{4} d \theta \\
\leq & -12 \int k^{2}\left(k^{\prime \prime}\right)^{2}\left(k^{\prime \prime \prime}\right)^{2} d \theta+24\left(\epsilon \int k^{2}\left(k^{\prime \prime}\right)^{2}\left(k^{\prime \prime \prime}\right)^{2}+1 / 4 \epsilon \int\left(k^{\prime}\right)^{2}\left(k^{\prime \prime}\right)^{4} d \theta\right) \\
& +36\left(\epsilon \int k^{2}\left(k^{\prime \prime}\right)^{2}\left(k^{\prime \prime \prime}\right)^{2}+1 / 4 \epsilon k^{2}\left(k^{\prime}\right)^{2}\left(k^{\prime \prime}\right)^{2} d \theta\right) \\
& -8 \alpha \int\left(k^{\prime}\right)^{2}\left(k^{\prime \prime}\right)^{3} d \theta-8 \alpha \int k\left(k^{\prime \prime}\right)^{4} d \theta \\
\leq & C_{1} \int\left(k^{\prime}\right)^{2}\left(k^{\prime \prime}\right)^{4} d \theta+C_{2} \int k^{2}\left(k^{\prime}\right)^{2}\left(k^{\prime \prime}\right)^{2} d \theta \\
& -8 \alpha \int\left(k^{\prime}\right)^{2}\left(k^{\prime \prime}\right)^{3} d \theta-8 \alpha \int k\left(k^{\prime \prime}\right)^{4} d \theta .
\end{aligned}
$$

By Young inequality, we have

$$
\begin{aligned}
& \int\left(k^{\prime}\right)^{2}\left(k^{\prime \prime}\right)^{3} d \theta \leq \epsilon \int\left(k^{\prime}\right)^{4 / 3}\left(k^{\prime \prime}\right)^{4} d \theta+C(\epsilon) \int\left(k^{\prime}\right)^{4} d \theta, \\
& \int k^{2}\left(k^{\prime}\right)^{2}\left(k^{\prime \prime}\right)^{2} d \theta \leq \epsilon \int\left(k^{\prime \prime}\right)^{4} d \theta+C(\epsilon) \int k^{4}\left(k^{\prime}\right)^{4} d \theta .
\end{aligned}
$$

Since $\left\|k^{\prime}\right\|_{\infty} \rightarrow 0$ as $t \rightarrow \infty$, we have

$$
\partial_{t} \int\left(k^{\prime \prime}\right)^{4} d \theta \leq-C_{1} \int\left(k^{\prime \prime}\right)^{4} d \theta+C_{2} \int\left(k^{\prime}\right)^{4} d \theta .
$$

Denote $\int\left(k^{\prime \prime}\right)^{4} d \theta$ by $f$. We have

$$
\partial_{t} f \leq-C_{1} f+C_{2} e^{-C_{3} t}
$$

By lemma 5.7.6 in [7], we complete the proof.

Lemma 37. There is some constant $C$ such that $\left\|k^{\prime \prime \prime}\right\|_{2} \leq C e^{-2 \beta t}$, where $\beta>0$ is a uniform constant.

Proof.

$$
\begin{aligned}
\partial_{t} \int\left(k^{\prime \prime \prime}\right)^{2} d \theta= & -2 \int k^{2}\left(k^{\prime \prime \prime \prime}\right)^{2} d \theta-8 \int k k^{\prime} k^{\prime \prime \prime} k^{\prime \prime \prime \prime} d \theta \\
& -4 \int\left(k^{\prime}\right)^{2} k^{\prime \prime} k^{\prime \prime \prime \prime} d \theta-4 \int k\left(k^{\prime \prime}\right)^{2} k^{\prime \prime \prime \prime} d \theta-6 \int k^{2} k^{\prime \prime} k^{\prime \prime \prime \prime} d \theta \\
& -12 \int k\left(k^{\prime}\right)^{2} k^{\prime \prime \prime \prime} d \theta-4 \alpha \int k\left(k^{\prime \prime \prime}\right)^{2} d \theta-12 \alpha \int k^{\prime} k^{\prime \prime} k^{\prime \prime \prime} d \theta .
\end{aligned}
$$


By Young inequality, we have

$$
\begin{aligned}
\partial_{t} \int\left(k^{\prime \prime \prime}\right)^{2} d \theta \leq & -2 \int k^{2}\left(k^{\prime \prime \prime \prime}\right)^{2} d \theta+\epsilon \int k^{2}\left(k^{\prime \prime \prime \prime}\right)^{2} d \theta+C_{1}(\epsilon) \int\left(k^{\prime}\right)^{2}\left(k^{\prime \prime \prime}\right)^{2} d \theta \\
& +C_{2}(\epsilon) \int \frac{\left(k^{\prime}\right)^{4}\left(k^{\prime \prime}\right)^{2}}{k^{2}} d \theta+C_{3}(\epsilon) \int\left(k^{\prime \prime}\right)^{4} d \theta \\
& +C_{4}(\epsilon) \int\left(k^{\prime}\right)^{4} d \theta+C_{5}(\epsilon) \int k^{2}\left(k^{\prime \prime}\right)^{2} d \theta-4 \alpha \int k\left(k^{\prime \prime \prime}\right)^{2} d \theta \\
& -12 \alpha\left(\epsilon \int\left(k^{\prime \prime \prime}\right)^{2} d \theta+C_{6}(\epsilon) \int\left(k^{\prime}\right)^{2}\left(k^{\prime \prime}\right)^{2} d \theta\right) .
\end{aligned}
$$

By the above estimate of $k^{\prime}$ and $k^{\prime \prime}$, for sufficiently large $t$, we have

$$
\partial_{t} \int\left(k^{\prime \prime \prime}\right)^{2} d \theta \leq-C_{6} \int\left(k^{\prime \prime \prime}\right)^{2} d \theta+C_{7} e^{-\beta t}
$$

The result follows from the lemma 5.7.5 in [7].

In fact, the method in 7 can be applied to our case. The high order estimate of $k$ is similar to [7, so we omit the detail.

\section{REFERENCES}

[1] Andrews,B., Evolving convex curves. Calc.Var.PDE's 7,315-371(1998).

[2] Bonnesen,T., Fenchel,W., Theorie der Convexen Körper.Chelsea,New York(1948).

[3] Chou,K.S., Zhu,X.P., The curve Shortening Problem. CRC Pre ss.Boca Raton (2001).

[4] Chow,B., Lu,P,Ni,L., Hamilton's Ricci Flow. Science Press/American Mathematical Society, Beijing/Providence(2006).

[5] Gage,M., An isoperimetric inequality with applications to curve shortening. Duke Math.J.50,1225-1229(1983).

[6] Gage, M.E., On an area-preserving evolution equation for plane curves. In: DeTurck, D.M. (ed.) Nonlinear Problems in Geometry, Contemp. Math, vol. 51, pp. 51 C62 (1986)

[7] Gage,M., R.S.Hamilton, The heat equation shrinking convex plane curves. J.Diff.Geom.,23,69-96(1986).

[8] Grayson,M., The heat equation shrinks embeded plane curves to round points. J.Diff.Geom.,26,285-314(1987).

[9] Huisken,G., Flow by mean curvature of convex surfaces into spheres. J.Diff.Geom.,20,237266(1984).

[10] Huisken, G., The volume preserving mean curvature flow. J. Reine Angew. Math. 382, 34-48 (1987).

[11] Jiang L.S., Pan, Shengliang, On a non-local curve evolution problem in the plane, Comm. Anal. Geom., 16(2008)1-26.

[12] Ma,L., D.Z.Chen, Curve shortening in a Riemannian manifold. Ann. Mat .Pura. Appl., 186,663-684(2007).

[13] Ma,L., Cheng, L., A non-local area preserving flow. Preprint, 2008.

[14] Osserman, R., Bonnesen isoperimetric inequalities. Amer.Math.Monthly 86(No.1)(1979).

[15] Perelman, G., Finite time extinction for the solutions to the Ricci flow on certain threemanifold. math.DG/0307245, 2003.

[16] Pan, ShengLiang, Junna Yang. On a non-local perimeter-preserving curve evolution problem for convex plane curves. manuscripta math.(2008).

[17] Tsai, D.H., Asymptotic closeness to limiting shapes for expanding embedded plane curves. Invent. Math. 162, 473C492 (2005)

Li Ma, Department of Mathematical Sciences, Tsinghua University, Beijing 100084, P. R. China

E-mail address: lma@math.tsinghua.edu.cn 